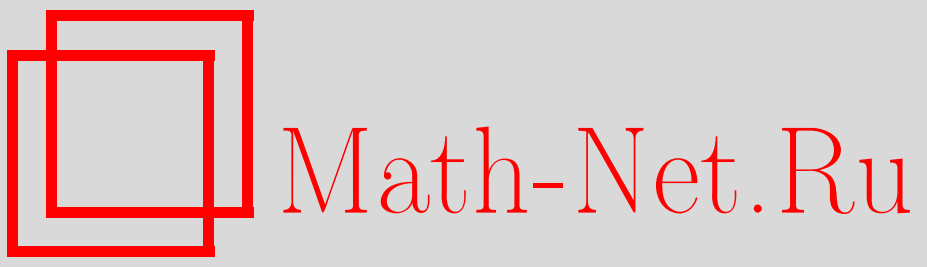

Ю. Брук, А. Стасенко, Объять необъятное, или Ее преПодобие Размерность, Квант, 2019, номер 2, 2-13

DOI: https://doi.org/10.4213/kvant20190201

Использование Общероссийского математического портала Math-Net.Ru подразумевает, что вы прочитали и согласны с пользовательским соглашением http://www.mathnet.ru/rus/agreement

Параметры загрузки:

IP : 54.198 .67 .100

26 апреля 2023 г., 10:25:50

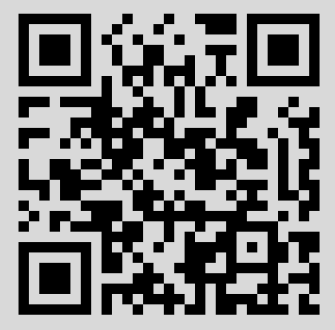




\section{Объять необъятное, или Ее преПодобие Размерность}

Ю.БРУК, А.СТАСЕНКО

\section{...необъятный океан истины простирается передо мною, уводя в неведомые дали.}

\section{Предварительные размышления}

О чем думает любознательный Пассажир авиалайнера, глядя через иллюминатор на изогнутое крыло самолета? Конечно же, о том, от каких параметров зависит подъемная сила, которая держит стотонную машину в воздухе. Прежде всего, Он может сообразить, что эта сила должна DOI: https://doi.org/10.4213/kvant20190201

\section{И.Ньютон}

расти с увеличением размера авиалайнера $l$, его скорости $v$ и плотности $\rho$ воздуха. А если при этом Он знает, в каких единицах выражается каждая из перечисленных физических величин, т.е. их размерность, то получит соотношение, уточняющее $x a$ рактер этой зависимости:

$$
F=l^{2} v^{2} \rho \cdot \operatorname{const}\left([\mathrm{F}]=\mathrm{H}=\frac{\mathrm{\kappa} \Gamma \cdot \mathrm{M}}{\mathrm{c}^{2}}\right) .
$$

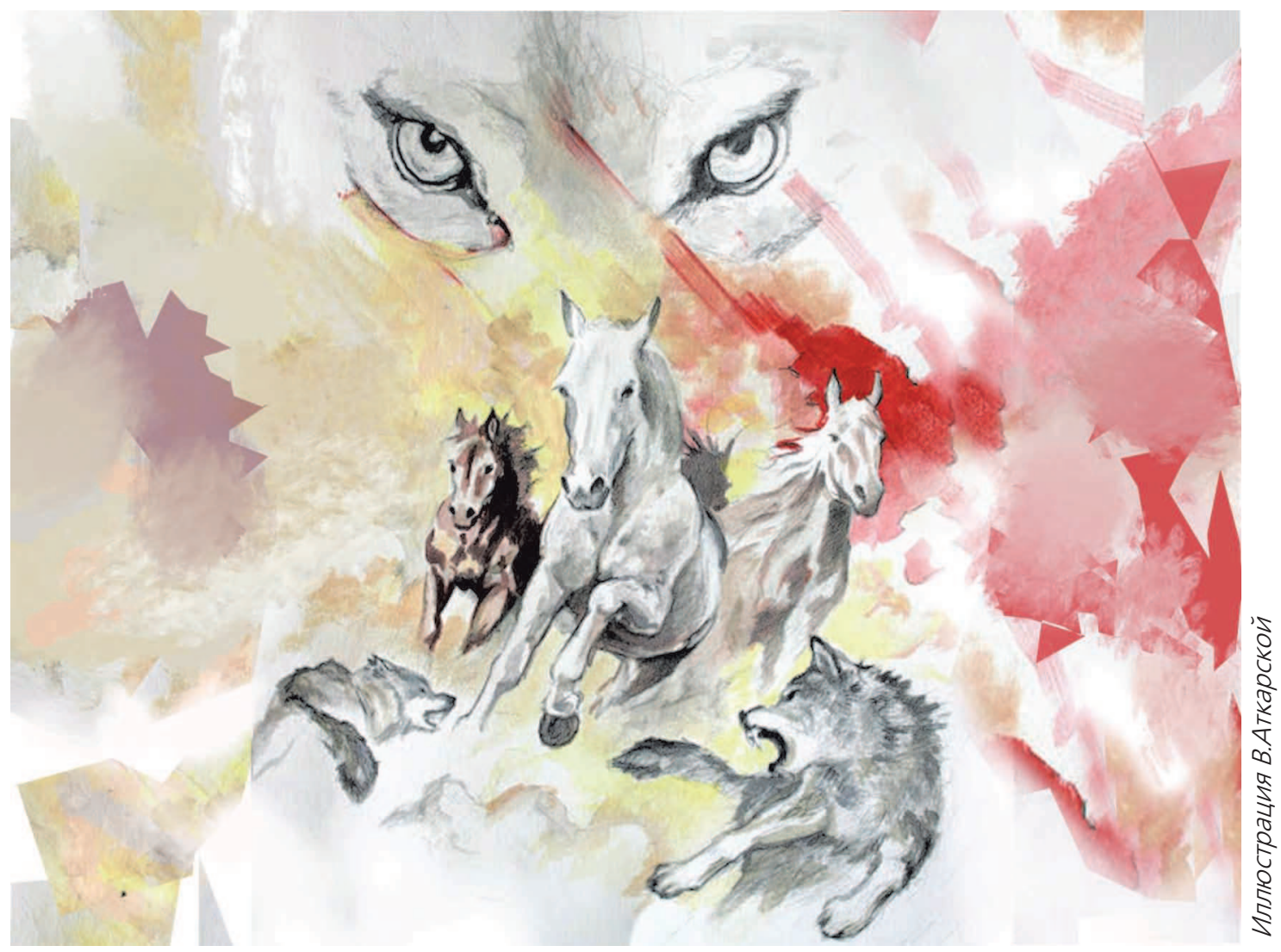


Здесь const - безразмерный множитель, зависящий от формы тела, обтекаемого воздухом, и от других параметров, ради определения которых во всем мире построены тысячи аэродинамических труб.

Какая польза от этого соотношения? Очень большая. Оно позволяет надеяться, что для тела заданной формы уже не нужно экспериментально «нащупывать» зависимость силы от всех определяющих параметров, а произвести лишь одно измерение на уменьшенной модели, геометрически подобной «большому» авиалайнеру.

Но что это - самолет начало слегка трясти? Это потому, что он летит в переохлажденном облаке - началось обледенение. Вспоминается знаменитый чкаловский перелет в Америку (1936 г.): «Первый контакт со стихией начинается над Кольским полуостровом... Появляются первые признаки обледенения - стекла пилотской кабины становятся матовыми. Начинается тряска...»

Как можно смоделировать в лабораторных условиях обтекание самолета воздухом, несущим водяные капли радиусом $a$ ? Заставить эту каплю обтекать тело радиусом $R$, когда она приобретает центростремительное ускорение $\frac{v^{2}}{R}$, может только аэродинамическая сила порядка $a^{2} v^{2} \rho$. Но поскольку масса капли $m \sim \rho_{0} a^{3}$, из равенства $\frac{m v^{2}}{R} \sim a^{2} v^{2} \rho$ следует

$$
\frac{a}{R} \sim \frac{\rho}{\rho_{0}} \sim 10^{-3},
$$

где $\rho_{0}-$ плотность воды. Это значит, что если в реальности $a=1 \mathrm{mм}=10^{-3} \mathrm{M}, R=$ $=1$ м, то на модели в сто раз меньших размеров нужно использовать капли в сто раз меньше, т.е. радиусом $10^{-5}$ м $=10$ мкм. «Конечно, все сложнее, но тут уже указана некая путеводная мысль»,- подумал любознательный Пассажир авиалайнера.

Здесь видна связь метода размерностей с важным физическим принципом, называемым принципом подобия. Фактически об этом принципе мы уже говорили выше.
Именно подобие позволяет нам моделировать различные физические явления.

В физике два явления называются подобными, если одинаковы безразмерные комбинации описывающих их величин. Перейти от описания одного явления к описанию другого, ему подобного, мы всегда можем простым пересчетом масштабов.

А о чем думали лилипуты, взирая на громаду Гулливера? Прежде всего - как ее прокормить. И придумали. Если считать, что тела лилипута и Гулливера не только геометрически подобны, но и одинаковы по своей структуре, то Гулливеру нужно пищи больше, чем лилипуту, во столько раз, во сколько раз отличаются объемы их тел, т.е. в $12^{3}=1728$ раз. Откуда взялось число 12 ? Надо отдать должное догадливости их ученых. Дело в том, что поверхность коры головного мозга лилипута в $12^{2}=144$ раза меньше; значит, и клеток серого вещества (ответственных за мышление) меньше во столько же раз (клетки не поддаются масштабированию). Впрочем, им помог сам Джонатан Свифт.

Но вот Гулливер попал в страну великанов, тоже в 12 раз больших ростом, чем он сам, по свидетельству автора, и столь же изящных. Возможно ли это? Ведь вес тела великана больше в $12^{3}$ раз, а сечение костей - всего в $12^{2}$ раз. А чтобы реализовать то же значение нагрузки на единицу площади поперечного сечения кости, диаметр сечения нужно увеличить еще в 12 раз! Тут уж не до изящества - вспомним слонов и мамонтов...

Еще Леонардо да Винчи «выводил из соображений подобия причину большого размера китов по сравнению со слонами: вес тела растет пропорционально кубу линейных размеров, прочность костей - пропорционально квадрату...» [1, с.73]. Он же «пытался понять законы газодинамики и турбулентности на основе степенны'х законов теории размерностей, явившихся предшественником колмогоровских» .

А вот еще один пример оценки, относящейся к «живой природе». Биологи утверждают, что только одна из десяти охотничьих экспедиций стаи голодных волков 
заканчивается успехом. Если стая гонится за табуном диких лошадей, то в $90 \%$ случаев табун уходит от волков без потерь. А ведь в нем, кроме взрослых лошадей, есть и маленькие жеребята. Значит, они бегают так же быстро, как и взрослые?

Рассмотрим взрослую лошадь и жеребенка. Будем думать, что сделаны они «из одного и того же мяса», т.е. примем, что, несмотря на различие в размерах, плотности их тел одинаковы. Пусть, кроме того, сила мышц в расчете на единицу поперечного сечения одна и та же. Обозначим через $M, L$, и $T$ массу, характерный размер и характерное время движения взрослого животного, а через $m, l$ и $t-$ те же величины для молодого. Тогда плотность и силу можно записать в соответствии с их размерностями в виде $\frac{M}{L^{3}}$ и $\frac{M L}{T^{2}}$ и, аналогично, $\frac{m}{l^{3}}$ и $\frac{m l}{t^{2}}$. Жеребенок и взрослая лошадь с достаточной точностью могут считаться геометрически подобными. Эти предположения дают теперь такие очевидные соотношения:

$$
\frac{\left(\frac{M}{L^{3}}\right)}{\left(\frac{m}{l^{3}}\right)} \sim 1, \frac{\left(\frac{M L}{T^{2}}\right): L^{2}}{\left(\frac{m l}{t^{2}}\right): l^{2}} \sim 1 .
$$

Отсюда получается, что скорости движения взрослой лошади и жеребенка одинаковы:

$$
\frac{L}{T} \sim \frac{l}{t} .
$$

Это объясняет, почему жеребенок в табуне лошадей, уходящих от волков, не отстает: он делает движения хотя и более мелкие, чем взрослая особь, но более частые.

\section{Нехитрый метод теории размерности}

Рассмотрим классическую задачу Рэлея. Пусть между точками $A$ и $B$ натянута струна, на середине которой находится шарик. Масса шарика $M$ намного больше массы самой струны. Струну оттягивают и отпускают. Пусть максимальное расстояние $x_{0}$ от шарика до прямой $A B$ мало по сравнению с длиной отрезка $A B$. Середину этого отрезка обозначим буквой $C$. При- мем еще, что $A C=C B=a$, движение шарика будем считать гармоническими колебаниями. Как будет зависеть частота этих колебаний $\omega$ от натяжения струны $F$, массы шарика $M$ и размера $a$ ?

Предположим, что величины $\omega, F, M$ и $a$ связаны степенно́й зависимостью:

$$
\omega \sim F^{x} M^{y} a^{z} .
$$

Здесь $x, y, z$ - некоторые числа, которые нам сейчас предстоит определить. Для этого выпишем размерности - наименования единиц, в которых измеряются интересующие нас величины в системе СИ:

$$
\begin{gathered}
{[\omega]=\mathrm{c}^{-1},[F]=H=\kappa \Gamma \cdot \mathrm{M} \cdot \mathrm{c}^{-2},} \\
{[M]=\kappa г,[a]=\mathrm{M}}
\end{gathered}
$$

(квадратные скобки как раз и обозначают размерность стоящей в них величины).

Мы не пишем в формулах, полученных таким методом, знак равенства, заменяя его волнистой чертой (знак пропорциональности). Следует иметь в виду, что метод размерностей не может помочь в вычислении численных коэффициентов в формулах.

Если написанная выше формула для $\omega$ выражает реальную физическую закономерность, то размерности правой и левой частей этой формулы должны совпадать. Поэтому можно записать такое равенство:

$$
\begin{array}{r}
\mathrm{c}^{-1}=\mathrm{H}^{x} \cdot \mathrm{K}^{y} \cdot \mathrm{M}^{z}=\mathrm{\kappa} \Gamma^{x} \cdot \mathrm{M}^{x} \cdot \mathrm{c}^{-2 x} \cdot \mathrm{K}^{y} \cdot \mathrm{M}^{z}= \\
=\mathrm{\kappa} \Gamma^{x+y} \cdot \mathrm{M}^{x+z} \cdot \mathrm{c}^{-2 x} .
\end{array}
$$

Очевидно, что должны удовлетворяться следующие уравнения для $x, y$ и $z$ :

$$
x+y=0, x+z=0,-2 x=-1 .
$$

Отсюда получаем

$$
x=1 / 2, \quad y=-1 / 2, z=-1 / 2,
$$

а значит,

$$
\omega \sim F^{1 / 2} M^{-1 / 2} a^{-1 / 2} .
$$

Интересно отметить, что точная формула для частоты отличается от найденной нами всего в $\sqrt{2}$ раз $\left(\omega^{2}=2 F /(M a)\right)$. Другими словами, можно в данном случае считать, что оценка для $\omega$ получена не только качественная (в смысле зависимости от параметров $F, M$ и $a$ ), но и количественная. По порядку величин найденная 
степенная комбинация $F, M$ и $a$ дает правильные значения частоты.

Нас и в дальнейшем часто будет интересовать оценка по порядку величины. В простых задачах обычно неопределяемые методом размерностей коэффициенты можно считать числами порядка единицы. Это, однако, не строгое правило. Окончательный вывод о величине численного коэффициента можно сделать, конечно, после сравнения с точной формулой либо из каких-то дополнительных соображений. Мы будем обсуждать подобные вопросы позже.

А сейчас стоит вернуться к задаче Рэлея и уточнить сделанные предположения. Вопервых, мы считали, что натяжение струны $F$ приблизительно одинаково, когда струна вытянута вдоль линии $A B$ и когда она оттянута перпендикулярно этой линии. Это предположение основано на том, что действительно существует связь между параметрами $\omega, F, M$ и $a$. Во-вторых, считалось, что формула, выражающая эту связь, имеет степенной вид: $\omega \sim F^{x} M^{y} a^{z}$.

Однако метод размерностей помогает находить функциональные зависимости между разными параметрами задачи не только для тех ситуаций, когда эти зависимости степенные. К счастью, таких зависимостей в природе довольно много, и метод размерностей становится хорошим помощником.

\section{Правило $\boldsymbol{N}-\boldsymbol{K}=1$}

Понятие размерности физической величины вводится тогда, когда уже выбраны некоторые основные физические величины и установлены единицы для их измерения. Величины, не включенные в число основных, называются производными величинами. Выражение единиц измерения производной физической величины через единицы измерения основных величин и означает размерность.

В общем случае выбор основных величин и единиц для их измерения может производиться разными способами. Здесь, конечно, многое зависит от удобства, традиций и существующих стандартов и соглашений. В механике основными величинами являются масса, длина и время.
Иногда говорят, что такие системы единиц принадлежат к классу $M L T$. Размерность силы в этом классе определяется выражением $M L T^{-2}$, размерность энергии $M L^{2} T^{-2}$ и т.д. Заменяя в этих выражениях $M$ на кг, $L$ на м, $T$ на с, мы получим размерности силы и энергии в системе СИ. Если мы рассматриваем задачи, в которых фигурируют немеханические величины, например электрический заряд, ток, потенциал, то можно увеличить число основных величин и соответствующих основных единиц измерения. В системе СИ в число основных величин добавляют силу тока, которую измеряют в амперах (А). Точно так же в термодинамике в число основных величин можно включить температуру, ее единица измерения - кельвин (К).

Важно отметить, что пользоваться методом размерностей можно в любой системе единиц. Каждый раз, конечно, выражения для размерностей различных величин нужно писать в одной заранее выбранной системе.

Предположим, что в какой-то задаче мы отыскиваем функциональную связь между $N$ величинами. Предполагая степенную зависимость одной из величин от других и выписывая размерности этих величин, мы можем пытаться построить интересующую нас формулу. Если все размерности выражаются через размерности $K$ основных величин и если при этом $N-K=1$, то можно утверждать, что искомая формула единственно возможная. Это правило наглядно иллюстрируется рассмотренным выше примером с колебаниями шарика на струне. Для этого случая у нас было четыре параметра: $\omega, T, M$ и $a$; размерности всех этих величин выражались через размерности основных величин, т.е. через кг, м, с. Другими словами, $N=4, K=3$ и $N-K=$ $=1$. То, что формула $\omega \sim T^{1 / 2} M^{-1 / 2} a^{-1 / 2}-$ единственно возможная, следует из того, что система уравнений для определения показателей степеней $x, y, z$ имеет единственное решение.

Рассмотрим теперь еще одну поучительную задачу - о колебаниях сферической капли. Простое решение этой задачи с помощью соображений размерности обсуж- 
далось Рэлеем еще в 1915 году. К этой задаче сводится, по существу, и вопрос о колебаниях атомных ядер, которые можно считать каплями ядерного вещества. Здесь мы обсудим сначала простейшую ситуацию.

Пусть из круглого отверстия вытекает сферическая капля. Поверхностная энергия при этом минимальна, а всякая система стремится попасть в состояние с минимальной энергией. Даже очень малые деформации капли приведут к тому, что силы поверхностного натяжения «заставят» ее пульсировать с некоторой частотой. При этом под пульсациями понимаются периодические изменения формы капли. Отвлечемся сейчас от вопроса, сколь быстро затухают подобные колебания. Нас будет интересовать частота (или период) процесса, которая может зависеть, очевидно, от коэффициента поверхностного натяжения $\sigma$, плотности жидкости $\rho$ и радиуса капли $a$. Запишем соотношение

$$
\omega \sim \sigma^{x} \rho^{y} a^{z}
$$

и попробуем найти числа $x, y, z$. Заметим, кстати, что под $\sigma$ можно понимать и плотность поверхностной энергии с размерностью $\frac{\text { Дж }}{\mathrm{m}^{2}}$, и силу, отнесенную к единице длины, размерность которой $\frac{\mathrm{H}}{\mathrm{M}}$. Выпишем размерности всех параметров:

$$
\begin{aligned}
& {[\omega]=\mathrm{c}^{-1},[\sigma]=\kappa \Gamma \cdot \mathrm{c}^{-2},} \\
& {[\rho]=\kappa \Gamma \cdot \mathrm{M}^{-3},[a]=\mathrm{M} .}
\end{aligned}
$$

Уравнения для определения $x, y, z$ получатся из равенства

$$
\mathrm{c}^{-1}=\kappa \Gamma^{x} \cdot \mathrm{c}^{-2 x} \cdot \kappa \Gamma^{y} \cdot \mathrm{M}^{-3 y} \cdot \mathrm{M}^{z} .
$$

Для трех неизвестных чисел есть три уравнения:

$$
-1=-2 x, x+y=0,-3 y+z=0 .
$$

Эта система уравнений имеет единственное решение:

$$
x=1 / 2, y=-1 / 2, z=-3 / 2,
$$

что находится в полном соответствии с правилом $N-K=1$.

Окончательно формула для частоты колебаний запишется так:

$$
\omega \sim\left(\frac{\sigma}{\rho a^{3}}\right)^{1 / 2} .
$$

Она подсказывает сразу же и принципиально возможный способ экспериментального определения величины $\sigma$. Для этого нужно знать плотность жидкости $\rho$, радиус капли $a$ и определить на опыте частоту $\omega$. То, что мы не знаем численного коэффициента в этой формуле, не должно быть серьезным препятствием. Можно, например, проделать опыт еще раз с жидкостью, для которой величина поверхностного натяжения известна, и после этого вычислить значения $\sigma$ для рассматриваемой жидкости.

По существу, мы сталкиваемся сейчас с простым случаем моделирования - колебания капли исследуемой жидкости можно моделировать колебаниями капли жидкости с известными $\sigma$ и $\rho$. В таких случаях и говорят о подобии физических явлений (в данном случае - колебаний формы капель) для двух разных жидкостей.

Еще одно любопытное замечание. Перепишем формулу для частоты колебаний так:

$$
\frac{\sigma}{\rho} \sim a^{3} \omega^{2} .
$$

Учтем теперь, что частота обратно пропорциональна периоду колебаний. Параметры $\sigma$ и $\rho$ характеризуют жидкость и потому одинаковы для любых капель этой жидкости. Пусть $T$ - период колебаний капельки, тогда $T^{2}=$ const $\cdot a^{3}$. Это важное утверждение. Если взять две капельки одной жидкости, но разных радиусов, то отношение квадратов периодов их колебаний будет равно отношению кубов их размеров. Как тут не вспомнить закон Кеплера для движения планет вокруг Солнца! Там, правда, нужно говорить не о радиусах шаров-планет, а о радиусах их орбит. Подумайте сами - нельзя ли извлечь что-нибудь полезное из этой $a н a-$ логии?

\section{А если $N-K>1 ?$}

Уже при выписывании системы параметров, связь между которыми мы хотим отыскать, нужно отдавать себе отчет в том, что существенно и что несущественно для конкретного физического явления. Если речь идет о динамике (например, о колеба- 
ниях), то в числе параметров должны быть силовая и массовая характеристики. Роль первой из них в предыдущем примере играла величина $\sigma$, роль второй - плотность жидкости $\rho$. В сущности, выше считалось, что колебания капли определяются поверхностным натяжением. Полученное решение, безусловно, годится, если капля колеблется в кабине космического корабля. А годится ли оно вблизи поверхности Земли? Не следует ли учесть еще и земное тяготение, другими словами вес капли?

Предположим, что мы захотели это сделать. Поступим сначала формально и запишем

$$
\omega \sim \sigma^{x} \rho^{y} a^{z} g^{t}
$$

где $g$ - ускорение свободного падения, $x$, $y, z$ и $t-$ неизвестные числа. Выписывая размерности всех величин, приходим к соотношению

$$
\mathrm{c}^{-1}=\mathrm{\kappa} \Gamma^{x} \cdot \mathrm{c}^{-2 x} \cdot \mathrm{\kappa}^{y} \cdot \mathrm{M}^{-3 y} \cdot \mathrm{M}^{z} \cdot \mathrm{M}^{t} \cdot \mathrm{c}^{-2 t} .
$$

Отсюда можно составить всего три уравнения, а неизвестных у нас - четыре. Решение такой системы уже не единственное, и возникает вопрос: как же теперь поступить? Было бы досадно, если бы было невозможно «выпутаться» из этого затруднения.

Попробуем рассуждать так. Зафиксируем пока число $x$, т.е. оставим его неопределенным (безразмерным!) параметром. Остальные неизвестные выразим через него:

$$
y=-x, z=-2 x-\frac{1}{2}, t=\frac{1}{2}-x .
$$

Тогда

$$
\omega \sim\left(\frac{g}{a}\right)^{1 / 2}\left(\frac{\sigma}{\rho a^{2} g}\right)^{x} .
$$

Именно для малых капель вес не должен быть определяющим параметром колебаний. Но тогда и ускорение $g$ не должно входить в формулу для $\omega$. Поэтому $x=1 / 2$. (Написанные равенства верны, разумеется, с точностью «по порядку величин». Неучтенные численные коэффициенты в этой задаче также порядка единицы.)

Мы вернулись к формуле, полученной раньше. Остается, возможно, все же не- которая неудовлетворенность тем, что пришлось прибегнуть к дополнительным аргументам и ограничиться частным случаем достаточно малых капелек. Про колебания капель (или больших шаров), когда нужно учитывать гравитацию, речь еще пойдет в дальнейшем. Пока же стоит подчеркнуть, что метод размерностей вовсе не есть универсальный инструмент для получения формул в физике. Он многое может, но не так уж редки и такие задачи, кода его разумно комбинировать с тем или иным способом, с другими идеями и методами.

\section{Метры «вдоль и поперек"}

В некоторых случаях удобно различать продольные и поперечные размеры тел и вводить различные единицы для измерения длин в разных направлениях. Этот прием позволяет увеличить число основных величин и часто оказывается полезным. Попробуем, например, оценить с помощью метода размерностей длину свободного пробега молекул в газе. Молекулы имеют конечные размеры и могут сталкиваться друг с другом даже в достаточно разреженных газах. Среднее расстояние, пробегаемое молекулами между двумя последовательными соударениями, и называется длиной свободного пробега. Обозначим ее буквой $l$, размер (радиус или диаметр) молекулы - буквой $a$, а концентрацию молекул в газе - буквой $n$. Молекулы условно считаются шариками, а под плотностью понимается число частиц в единичном объеме.

Размерность $n$ есть, очевидно, м $^{-3}$, размерности $l$ и $a-$ это м. Основная величина в данном случае одна - длина. Параметров, связь между которыми мы хотим найти, три: $l, a$ и $n$. Разность $N-K=2$. С точки зрения размерностей устраивают в качестве допустимых любые комбинации вида

$$
l \sim a, l \sim n^{-1 / 3}, l \sim\left(n a^{2}\right)^{-1}, l \sim a^{3} n^{2 / 3}, \ldots
$$

Какую же из них выбрать? Неужели метод размерностей не поможет найти правильную формулу? Это было бы тем долее обидно, что задача-то ведь совсем простая, 
а метод размерностей «работает», как мы знаем, и в более сложных случаях. Вот здесь-то и поможет упомянутый в начале этого раздела прием.

Условимся следить за какой-нибудь одной молекулой. «Привяжем» к ней систему прямоугольных координат, причем одну из осей направим вдоль траектории молекулы. Предполагается, что в первом приближении между двумя последовательными столкновениями молекула летит по прямой линии. Длину пробега естественно измерять в «продольных» метрах - $\mathbf{M}_{\|}$. Поперечные размеры молекул, мешающих движению нашей избранницы, будем измерять, соответственно, в «поперечных» метрах $-\mathrm{m}_{\perp}$. А как быть с концентрацией? Какие метры выбрать здесь? Считая, что направления, поперечные по отношению к траектории молекулы, эквивалентны, легко сообразить, что объем произвольного, в том числе и единичного, кубика, построенного на наших координатных осях, естественно измерять в $\mathrm{M}_{\|} \mathrm{M}_{\perp}^{2}$. Но тогда размерность числа частиц в единичном объеме $(n)$ будет $\mathbf{M}_{\|}^{-1} \mathrm{M}_{\perp}^{-2}$, а размерностью поперечных сечений молекул, мешающих интересующему нас движению, будет $\left[a_{\perp}^{2}\right]=\mathrm{m}_{\perp}^{2}$.

После всех этих ухищрений из множества выписанных выше возможных зависимостей $l$ от $n$ и $a$ совершенно однозначно остается единственная формула:

$$
l \sim n^{-1} a^{-2} .
$$

«Расслоив» метры на «параллельные» и «перпендикулярные», мы увеличили тем самым число основных величин, их стало две, и теперь разность $N-K$ снова равна единице.

Введенные единицы носят название направленных или векторных единиц длины. Чтобы получше освоиться с ними, вернемся к уже обсуждавшейся выше задаче о колебаниях капли. Предположим снова, что частота колебаний может быть связана с величиной поверхностного натяжения $\sigma$, плотностью $\rho$, размером капли $a$ и ускорением свободного падения $g$. Выпишем размерности основных величин: кг, с, $\mathbf{M}_{\|}, \mathrm{M}_{\perp}$. В задаче есть выделенное направление - оно задается вектором $\vec{g}$, можно назвать его «продольным», тогда $[g]=\mathrm{M}_{\|} \cdot \mathrm{c}^{-2}$. Объем капли будет измеряться в единицах $\mathrm{m}_{\|} \mathrm{m}_{\perp}^{2}$, размерность радиуса капли $a$ при этом будет $\mathrm{m}_{\|}^{1 / 3} \mathrm{M}_{\perp}^{2 / 3}$. Размерность же плотности $\rho$ будет равна кг $\cdot \mathbf{M}_{\|}^{-1} \mathbf{M}_{\perp}^{-2}$, а размерность $\sigma$ будет $\frac{\kappa \Gamma \cdot \mathrm{M}_{\|}}{\mathrm{c}^{2} \cdot \mathrm{M}_{\|}}=\kappa \Gamma \cdot \mathrm{c}^{-2}$. То, что размерность $\sigma$ содержит именно «продольные» метры $\mathrm{M}_{\|}$, нетрудно понять, вспомнив, как определяется поверхностное натяжение. Рассмотрим мыльную пленку, натянутую на проволочную рамку. Пленка сопротивляется растяжению в том направлении, в котором ее тянут. Ускорение, как уже договорились, измеряем в $\mathrm{m}_{\|} \cdot \mathrm{c}^{-2}$, значит, размерность силы - это кг $\cdot \mathrm{M}_{\|} \cdot \mathrm{c}^{-2}$. Формула $\omega \sim \sigma^{x} \rho^{y} a^{z} g^{t}$ приводит теперь к равенству

$$
\begin{aligned}
\mathrm{c}^{-1}=\mathrm{\kappa} \Gamma^{x} \cdot \mathrm{c}^{-2 x} \cdot \mathrm{\kappa} \Gamma^{y} & \cdot \mathrm{M}_{\|}^{-y} \cdot \mathrm{M}_{\perp}^{-2 y} \times \\
& \times \mathrm{M}_{\|}^{z / 3} \cdot \mathrm{M}_{\perp}^{2 z / 3} \cdot \mathrm{M}_{\|}^{t} \cdot \mathrm{c}^{-2 t} .
\end{aligned}
$$

Правило $N-K=1$ выполняется: $N=5$, $K=4$. Система линейных уравнений для определения $x, y, z$ и $t$ такова:

$$
\begin{aligned}
-1=-2 x-2 t, 0 & =x+y, 0=-y+\frac{z}{3}+t, \\
0 & =-2 y+\frac{2 z}{3} .
\end{aligned}
$$

Единственное решение этой системы:

$$
x=1 / 2, y=-1 / 2, z=-3 / 2, t=0 .
$$

Формула для частоты колебаний капли, конечно, прежняя:

$$
\omega \sim\left(\frac{\sigma}{\rho a^{3}}\right)^{1 / 2} .
$$

То, что из формулы «выпало» ускорение $g$, означает, что первоначальное предположение о возможной зависимости от $g$ оказалось несправедливым. Метод размерностей позволяет, таким образом, исключать лишние параметры.

Но мы как будто не использовали сейчас предположение о малости капель? А ведь раньше говорилось о том, что полученная формула для $\omega$ годится только при описании достаточно малых капелек. И критерием малости было неравенство 
$\sigma a \gg \rho a^{3} g$. Перепишем его в виде

$$
\left(\frac{a}{g}\right)^{1 / 2}>\left(\frac{\rho a^{3}}{\sigma}\right)^{1 / 2}
$$

и заметим, что слева стоит время падения капли на расстояние порядка ее размеров, а справа - период колебаний $\left(\sim \omega^{-1}\right)$. Это последнее неравенство означает, что колебания, обусловленные силами поверхностного натяжения, можно рассматривать на фоне относительно медленного падения капли. Разница характерного времени падения (на расстояние $\sim a$ ) и периода колебаний позволяет разделить эти два процесса и рассматривать их независимо. Поэтому в нашем примере период колебаний и на самом деле не зависит от $g$.

Сделаем еще несколько замечаний о векторных единицах длины. Во-первых, существуют задачи, в которых целесообразно вводить не только «продольные» и «поперечные» размеры, но и разные силы (одни, измеряемые в кг $\mathrm{m}_{\|} \cdot \mathrm{c}^{-2}$, другие - в кг $\left.\mathrm{M}_{\perp} \cdot \mathrm{c}^{-2}\right)$. Во-вторых, возможно естественное обобщение идеи о введении «своих единиц» для измерения продольных и поперечных размеров. В случае необходимости в какой-то конкретной задаче можно было бы ввести «свои метры» для каждого из трех взаимно-перпендикулярных направлений в пространстве. Число основных единиц, таким образом, увеличивается сразу на две. Третье замечание такое. Бывает так, что две существенно различные физические величины имеют в какой-то системе единиц одинаковые размерности. В системе СИ, например, такими величинами являются момент силы $L$ и работа $A$. Обе величины имеют размерности кг $\cdot \mathrm{m}^{2} \cdot \mathrm{c}^{-2}$. Обычный метод размерностей не может эти величины различить. Но если мы снова применим нашу «маленькую хитрость», введя разные единицы длины вдоль траектории движения и перпендикулярно к ней, то $L$ и $A$ сразу станут различаться. При вычислении работы всегда проектируется сила на направление движения. В определении момента силы существенны «перпендикулярные» единицы длины.
Другими словами, момент есть векторное, а работа - скалярное произведение: $\vec{L}=\vec{F} \times \vec{r}, \quad A=\vec{F} \cdot \vec{r}$, где $\vec{F}-$ сила, $\vec{r}-$ радиус-вектор. Ясно еще, что размерность самой силы содержит «продольные» метры. Тогда получится, что $L$ имеет размерность кг $\cdot \mathrm{M}_{\|} \cdot \mathrm{M}_{\perp} \cdot \mathrm{c}^{-2}, A-$ размерность $\mathrm{\kappa} \Gamma \cdot \mathrm{M}_{\|}^{2} \cdot \mathrm{c}^{-2}$.

\section{Колебания формы капли}

Из всего сказанного выше ясно, что колебания капли происходят в результате противодействий поверхностного натяжения, характеризуемого коэффициентом $\sigma$ (жесткость), которое стремится восстановить шаровую форму, и инертности, измеряемой массой $m$, заставляющей проскакивать положение равновесия, так что

$$
\omega_{0} \sim \sqrt{\frac{\sigma}{m}} \sim \sqrt{\frac{\sigma}{\rho a^{3}}} .
$$

Например, для капли дождя радиусом $a=$ $=1$ мм получим оценку

$$
\omega_{0} \sim \sqrt{\frac{0,06}{10^{3} \cdot 10^{-9}}} c^{-1} \approx 250 \mathrm{c}^{-1} .
$$

\section{Колебания формы атомного ядра (капельная модель)}

Ядро с атомным номером $A$ содержит число протонов и нейтронов, в сумме равное $A$. Принимая энергию связи равной $E_{1}=8,5 \mathrm{MэВ/нуклон,} \mathrm{полную} \mathrm{энергию}$ связи оценим как $A E_{1}$ и, «приписывая» ее поверхностному натяжению, найдем коэффициент $\sigma=\frac{A E_{1}}{4 \pi a^{2}}$, где $a=r_{p} \sqrt[1 / 3]{A}-$ радиус ядра-капли, $r_{p}$ - радиус нуклона (протона). Тогда частота колебаний будет порядка

$$
\begin{aligned}
\omega_{0} \sim\left(\frac{E_{1}}{4 \pi a^{2} m_{p}}\right)^{1 / 2} \sim\left(\frac{E_{1}}{4 \pi A^{1 / 3} r_{p}^{2} m_{p}}\right)^{1 / 2} \sim \\
\sim 4 \cdot 10^{21} \mathrm{c}^{-1}
\end{aligned}
$$

( здесь принято $m_{p}=1,67 \cdot 10^{-27}$ кг, $r_{p} \sim 10^{-15} \mathrm{м}, A=235$ для ядра $\left.{ }_{92}^{235} \mathrm{U}\right)$. Такая частота характерна для $\gamma$-излучения. 


\section{Колебания формы Земли}

Если Земной шар уподобить капле, то гравитационная энергия, «размазанная» по ее поверхности, будет порядка $\sigma \sim \frac{G M^{2}}{4 R \pi R^{2}}$, откуда $\omega_{0} \sim \sqrt{\frac{\sigma}{M}} \sim \sqrt{G \rho}$, где $G=6,67 \cdot 10^{-11} \mathrm{H} \cdot \mathrm{м}^{2} / \kappa^{2}-$ постоянная всемирного тяготения. Считая $\rho \sim 5,5$ г $/ \mathrm{cm}^{3}$, получим

$$
\omega_{0} \sim 6 \cdot 10^{-4} \mathrm{c}^{-1} \text {, или } T_{0}=\frac{2 \pi}{\omega_{0}} \sim 3 \text { часа . }
$$

\section{Колебания Вселенной}

Принимая среднюю плотность Вселенной равной $\rho_{\mathrm{cp}} \sim 5 \cdot 10^{-30} \mathrm{r} / \mathrm{cm}^{3}$ (критическое значение, разделяющее колебательный и монотонный характеры ее динамики), для периода колебаний получим

$$
T_{0} \sim(G \rho)^{-1 / 2} \sim 10^{18} \mathrm{c} \sim 3 \cdot 10^{10} \text { лет . }
$$

(В настоящее время Вселенная расширяется, а ее дальнейшая судьба, согласно космологии, зависит от того, что больше: средняя или критическая плотность.)

Астрономические наблюдения смещения линий излучения показали, что галактики удаляются от нас со скоростью, пропорциональной расстоянию: $V=H r$ (чем дальше наблюдаемая галактика, тем она «краснее»). Эта ситуация аналогична распределению скоростей в случае точечного взрыва. Коэффициент пропорциональности это постоянная Хаббла $H=2,2 \cdot 10^{-18}$ с (но обычно она выражается в км/с на млн световых лет). Интересно отметить, что обратная величина $\tau=\frac{1}{H}>10$ млрд лет имеет смысл периода колебаний Вселенной, а величина $\frac{c}{H} \sim 10^{26}$ м - размеров Вселенной.

В частности, третий закон Кеплера можно считать одним из примеров подобия. Действительно, записав закон всемирного тяготения Ньютона в безразмерном виде, отнеся расстояние $r$ планеты от Солнца к большой полуоси $a$ ее эллиптической траектории, а соответствующий момент времени $t-$ к периоду ее обращения $T$, получим

$$
\frac{d^{2}\left(\frac{r}{a}\right)}{d\left(\frac{t}{T}\right)^{2}}\left(\frac{r}{a}\right)^{2}=G M_{\mathrm{C}} \frac{T^{2}}{a^{3}} .
$$

Так как $G$ и $M_{\mathrm{C}}$ (масса Солнца) - постоянные, следует, что $T^{2} \sim a^{3}$ для всех планет нашей системы.

\section{Гравитационная постоянная и скорость света}

Продолжим обсуждение вопроса о том, в каких случаях следует ожидать появления в формулах размерных постоянных. В этом разделе речь пойдет о гравитационной постоянной $G$ и скорости света $c$.

Естественно, что $G$ появляется в задачах, описывающих гравитационное взаимодействие, а $c$ - в задачах, где речь идет о движении частиц со скоростями, близкими к скорости света. Присутствует скорость света и в задачах, относящихся к распространению света и электромагнитного излучения - такое излучение есть просто поток фотонов, а они всегда, конечно, движутся со скоростью света.

Пример, который мы теперь рассмотрим, требует одновременного включения в число определяющих параметров обеих констант $G$ и $c$. Сейчас хорошо известно, что луч света, идущий из далекой галактики и проходящий вблизи края солнечного диска, отклоняется на малый угол $\varphi_{\mathrm{C}} \approx 2 \cdot 10^{-6}$ рад . Известны масса Солнца $M_{\mathrm{C}} \simeq 2 \cdot 10^{30}$ кг и его радиус $R_{\mathrm{C}} \simeq 7 \cdot 10^{5}$ км. Гравитационное поле Солнца служит причиной отклонения светового луча, само же это поле определяется параметрами $G, M_{\mathrm{C}}$ и $R_{\mathrm{C}}$. Интересно получить формулу, связывающую угол $\varphi_{\mathrm{C}}$ с параметрами $G, M_{\mathrm{C}}, R_{\mathrm{C}}$ и $c$. Никаких других параметров в задаче нет. Говоря, что луч света проходит вблизи звезды, мы пренебрегаем, конечно, разницей между радиусом звезды и расстоянием от ее центра до ближайшей точки луча. Запишем, как обычно, формулу

$$
\varphi_{\mathrm{C}} \sim M_{\mathrm{C}}^{x} R_{\mathrm{C}}^{y} G^{z} c^{t} .
$$

Размерность гравитационной постоянной 
проще всего получить из закона всемирного тяготения:

$$
[G]=\frac{[F]\left[r^{2}\right]}{\left[m^{2}\right]}=\mathrm{H} \cdot \mathrm{M}^{2} \cdot \mathrm{K}^{-2}=\mathrm{K}^{-1} \cdot \mathrm{M}^{3} \cdot \mathrm{c}^{-2} \text {. }
$$

Угол же $\varphi_{\mathrm{C}}-$ величина безразмерная, поэтому мы получим три таких уравнения:

$$
x-z=0, y+3 z+t=0,-2 z-t=0 .
$$

Неизвестных же чисел - четыре, правило $N-K=1$ не выполнено. Но с подобной ситуацией мы уже сталкивались и знаем, что теперь нужна какая-то дополнительная информация. Зафиксируем число $x$, тогда $y=-x, z=x, t=-2 x$. Интересующая нас формула может быть представлена в виде

$$
\varphi_{\mathrm{C}} \sim\left(\frac{G M_{\mathrm{C}}}{R_{\mathrm{C}} c^{2}}\right)^{x} .
$$

Для определения числа $x$ можно использовать теперь известное из наблюдений и приведенное выше численное значение $\varphi_{\mathrm{C}}$. Учитывая, что $G \simeq 6,67 \cdot 10^{-11} \mathrm{H} \cdot \mathrm{m}^{2} / \mathrm{\kappa г}^{2}$, а скорость света $c \simeq 3 \cdot 10^{8} \mathrm{~m} / \mathrm{c}$, легко проверить, что написанная формула правильна при $x=1$.

Соображения подобия позволяют считать, что угол отклонения светового луча в гравитационном поле любой звезды с массой $M$ и радиусом $R$ определяется аналогичным образом и равен $\varphi \sim \frac{G M}{R c^{2}}$.

В этих рассуждениях появились два новых соображения. Прежде всего, мы не можем утверждать только на основании сказанного выше, что $x=1$. Формулы выписаны только по порядку величины, и, казалось бы, ничто не мешает нам считать, что $x=0,99$ или $x=1,02$. Уверенность в том, что $x=1$ основана не на приведенных формальных вычислениях, да и к тому же с неизвестным численным коэффициентом и с приближенными значениями всех параметров, а на опыте и на соображениях уже не размерности, а «разумности». Опыт говорит нам, что показатели степеней в формулах бывают обычно целыми числами или простыми дробями. В то же время не видно каких-либо оснований для того, чтобы считать, например, $x=8 / 9$ или $x=19 / 21$. «Разумность» и желание видеть формулы возможно более изящными играют вовсе не последнюю роль в физике! Нельзя, конечно, целиком отдаваться эмоциям, но ничего другого без точного решения обсуждаемой задачи мы все равно сказать не смогли бы. Точное же решение на самом деле приводит к $x=1$.

Второе обстоятельство заключается в том, что безразмерная комбинация параметров $M, R, G$ и $c$ приводит для большого числа звезд к значению $\varphi \ll 1$. Обратите внимание на то, что $\varphi$ сейчас не является безразмерным постоянным коэффициентом, это - функция других параметров, функция безразмерная, но вовсе не обязанная быть универсальной константой! Неопределяемая же из соображений размерности константа в правой части формулы $\varphi \sim \frac{G M}{R c^{2}}$, конечно, тоже есть, и вот она-то порядка единицы.

Малость значения функции $\varphi$ для Солнца вовсе не означает, что эта функция всегда мала. Пользуясь полученной для нее формулой, можно оценить отклонение луча света, проходящего вблизи нейтронных звезд. Так называют звезды, плотность которых примерно такая же, как плотность вещества внутриатомных ядер. Их массы по порядку величины совпадают с массой нашего Солнца, а радиусы составляют всего лишь десятки километров. Гравитационное поле нейтронных звезд намного «сильнее» поля Солнца, поэтому неудивительно, что типичное значение угла $\varphi$ для них достигает уже десятых долей радиана! Читатель легко проверит это сам.

\section{Три фундаментальные константы}

Фундаментальными (или мировыми) константами часто называют гравитационную постоянную $G$, скорость света $c$ и постоянную Планка $h$, входящую в формулы, описывающие квантовые явления.

Наш читатель уже знает, вероятно, что излучаемая (или поглощаемая) атомами энергия квантуется. В простейшей квантовой модели атом рассматривается по аналогии с планетной системой. Электроны 
вращаются вокруг ядра по орбитам вполне определенных радиусов, переход же электрона с одной орбиты на другую должен сопровождаться излучением или поглощением кванта энергии величиной $h v$. Здесь $v$ - частота излучения, определяемая разностью энергий электрона на соответствующих орбитах. Отсюда ясно, что размерность $h$ в системе СИ определяется так:

$$
[h]=\text { Дж } \cdot \mathrm{c}=\kappa г \cdot \mathrm{m}^{2} \cdot \mathrm{c}^{-1} .
$$

Теперь можно обсудить, например, вопрос об излучении энергии нагретым телом. Такое тело излучает обычно энергию в разных диапазонах. Потоки тепла, света и рентгеновского излучения можно рассматривать, конечно, как потоки квантов соответствующих частот.

Пусть температура излучающей поверхности равна $T$. Обозначим буквой $q$ энергию, излучаемую с единицы поверхности за единицу времени. Эта величина имеет смысл плотности потока энергии, ее размерность в системе СИ есть $[q]=\frac{\text { Дж }}{\mathrm{M}^{2} \cdot \mathrm{c}}=\kappa \Gamma \cdot \mathrm{c}^{-3}$. Кванты электромагнитного излучения движутся со скоростью $c$. Отвечающая температуре $T$ характерная энергия есть, очевидно, $k T$, где $k$ - постоянная Больцмана. Естественно предположить, что функционально могут быть связаны четыре величины: $q,(k T)$, $c$ и $h$. Из соображений размерности тогда легко получить формулу $q \sim(k T)^{4} c^{-2} h^{-3}$. Часто этот закон записывают в виде $q=\sigma T^{4}$ и называют законом СтефанаБольцмана. Коэффициент $\sigma$ называется, соответственно, постоянной СтефанаБольцмана. Ясно, что в системе СИ $[\sigma]=\kappa г \cdot \mathrm{c}^{-3} \cdot \mathrm{K}^{-4}$. Приведем еще для полноты численные значения констант, входящих в написанные формулы:

$$
\begin{gathered}
k=1,38 \cdot 10^{-23} \text { Дж/К, } \\
h=6,63 \cdot 10^{-34} \mathrm{\kappa г} \cdot \mathrm{M}^{2} \cdot \mathrm{c}^{-1} .
\end{gathered}
$$

Таким образом, мы познакомились с несколькими размерными константами. Формулы для $q$ и $\sigma$ содержат две фундаментальные константы: $c$ и $h$. Это есть прямое указание на то, что строгое реше- ние задачи об излучении нужно строить на основе квантовой и одновременно релятивистской теорий. Постоянная СтефанаБольцмана, вычисляемая в такой теории, равна $\sigma=\frac{\pi^{2} k^{4}}{60 h^{3} c^{2}}=5,67 \cdot 10^{-8} \kappa \Gamma \cdot \mathrm{c}^{-3} \cdot \mathrm{K}^{-4}$. Зная этот закон, можно довольно просто оценить температуру $T_{\mathrm{C}}$ «поверхности» Солнца площадью $4 \pi R_{\mathrm{C}}^{2}$, используя уравнение баланса тепла, поглощаемого диаметральным сечением Земли $\pi R_{3}^{2}$ и излучаемого с ее поверхности $4 \pi R_{3}^{2}$ при известной средней температуре $T_{3} \approx 300 \mathrm{~K}$ :

$$
\frac{4 \pi R_{\mathrm{C}}^{2} \sigma T_{\mathrm{C}}^{4}}{4 \pi l_{\mathrm{C} 3}^{2}} \pi R_{3}^{2}=4 \pi R_{3}^{2} \sigma T_{3}^{4},
$$

откуда

$T_{\mathrm{C}}=T_{3} \cdot(4 \cdot 4)^{1 / 4} \cdot\left(\frac{l_{\mathrm{C} 3}}{2 R_{\mathrm{C}}}\right)^{1 / 2}=20 T_{3}=6000 \mathrm{~K}$.

Здесь $l_{\text {Сз }}$ - расстояние от Земли до Солнца, а $\frac{2 R_{\mathrm{C}}}{l_{\mathrm{C} 3}} \approx 10^{-2}$ рад - угол, под которым диаметр Солнца виден с Земли. При этом нам не пришлось лететь к Солнцу с термометром. Более того, оказалось необязательно знать и $\sigma$, а нужно знать только температурную зависимость плотности потока излучения.

Не случайно академик Н.Н.Боголюбов «всегда ценил умение быстро прикинуть ответ на трамвайном билете» [1, с.196].

А вот исторический факт. «Капица был великим инженером и замечательным физиком... я помню то впечатление, которое произвела на нас сделанная им оценка мощности взрыва первой бомбы в Хиросиме... Какой-то... журналист... заметил, что на некоей железнодорожной станции... невдалеке от Хиросимы... аккуратно вывалились написанные черной тушью иероглифы... Белая часть бумажной поверхности объявления никак не пострадала... Исходя из того, что окрашенная часть мишени поглощает все, а белая отражает, Петр Леонидович... вычислил равновесную температуру в области взрыва, что и позволило оценить его мощность... П.Л.Капица показал нам... всю мощь метода оценок порядка величин исходя из здравого 
смысла и простых физических соображений (что на самом деле, заметим в скобках, одно и то же)» $[2$, с.230].

Отметим, кстати, что численный коэффициент $\frac{\pi^{2}}{60}$ в приведенной выше формуле для $\sigma$ тоже можно считать при грубых оценках числом порядка единицы.

Зададим теперь такой вопрос: в каком случае в формулах могут содержаться все три фундаментальные константы? Очевидно, что для этого существенными должны быть одновременно гравитация, релятивизм и квантование. Любопытно, что из $G, c$ и $h$ можно сконструировать величины с размерностями массы, длины и времени. Из соображений размерности такие соотношения строятся сразу же и однозначно и имеют такой вид:

$$
\begin{gathered}
m_{P} \sim\left(\frac{h c}{G}\right)^{1 / 2}, l_{P} \sim\left(\frac{G h}{c^{3}}\right)^{1 / 2}, \\
t_{P} \sim\left(\frac{G h}{c^{5}}\right)^{1 / 2} .
\end{gathered}
$$

Если принять неопределенные численные коэффициенты в этих формулах равными единице, то получаются «естественные» масштабы для измерения масс, длин и времени. Эти масштабы впервые ввел М.Планк, и они называются планковскими единицами. Нетрудно проверить, что

$$
\begin{gathered}
m_{P} \sim 2 \cdot 10^{-8} \text { кг }, l_{P} \sim 2 \cdot 10^{-35} \mathrm{M} \\
\text { и } t_{P} \sim 5 \cdot 10^{-44} \mathrm{c} .
\end{gathered}
$$

Для практических задач, возникающих в повседневной жизни, это слишком малые масштабы. Современная физическая теория, однако, не может пренебрегать и такими масштабами. В космологии - науке, описывающей эволюцию Вселенной, планковский период играет очень важную роль, именно планковские масштабы определяют самую раннюю историю нашего Мира. Строгой теории физических процессов, происходивших на начальных стадиях жизни Вселенной, пока еще не существует. Никаких экспериментов со Вселенной (кроме, быть может, мысленных) мы проводить тоже не можем. Но уже сама возможность построения планковских еди- ниц показывает нам, что будущая теория ранней Вселенной должна быть, во всяком случае, квантовой гравитационной теорией.

Чтобы лишний раз проиллюстрировать, сколь непросто построить такую теорию, вычислим еще планковские единицы плотности и температуры:

$$
\begin{gathered}
\rho_{P} \sim \frac{m_{P}}{l_{P}^{3}} \sim \frac{c^{5}}{G^{2} h}=5 \cdot 10^{96} \mathrm{\kappa г} / \mathrm{M}^{3}, \\
T_{P} \sim \frac{1}{k}\left(\frac{h c^{5}}{G}\right)^{1 / 2}=10^{12} \mathrm{~K} .
\end{gathered}
$$

Вероятно, эти грандиозные численные значения должны заставить читателя окончательно поверить в то, что свойства вещества или излучения, характеризующиеся такими параметрами, не могут быть простыми и, скорее всего, вовсе не похожи на все то, к чему мы привыкли.

Итак, рассмотренные примеры использования метода размерностей и подобия позволяют определить характер зависимости между величинами с точностью до безразмерных множителей, но зато какой диапазон - от атомного ядра до Вселенной!

Завершим словами замечательных ученых об удивительно плодотворном физико-математическом синтезе:

«...слияние математики и физики заставляет... вспоминать о том времени, когда представители точных наук именовались просто натурфилософами» [3, с.34].

«Это более мощный инструмент познания, чем все остальное, что дала нам человеческая деятельность... рассматривается либо порядок, либо мера и совершенно несущественно, будут ли это числа, фигуры, звезды, звуки или что-нибудь другое» [4, с.58].

\section{Литература}

1. В.И.Арнольд. К восьмидесятилетию. М.: МЦНМО, 2018.

2. Н.В.Карлов. Книга о Московском Физтехе. -М.: ФИЗМАТЛИТ, 2008.

3. Н.Н.Боголюбов. Собрание научных трудов. Т.1. - М.: Наука, 2005.

4. Р.Декарт. Правила для руководства ума. - М.-Л.: Соцэкгиз, 1936. 